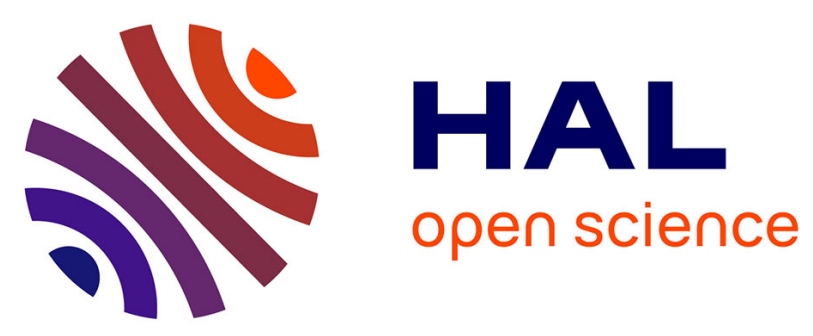

\title{
All-optical phase-preserving amplitude regeneration of 28-Gbaud RZ-DQPSK signals with a microcavity saturable absorber in a recirculating loop experiment
}

Quang Trung Le, Laurent Bramerie, Mathilde Gay, Michel Joindot, Jean-Claude Simon, Arthur O’Hare, Hoang Trung Nguyen, Jean-Louis Oudar

\section{- To cite this version:}

Quang Trung Le, Laurent Bramerie, Mathilde Gay, Michel Joindot, Jean-Claude Simon, et al.. Alloptical phase-preserving amplitude regeneration of 28-Gbaud RZ-DQPSK signals with a microcavity saturable absorber in a recirculating loop experiment. Optical Fiber Communication conference and National Fiber Optic Engineers Conference 2011 (OFC/NFOEC 2011), Mar 2011, Los Angeles, CA, United States. pp.OMT5, 10.1364/OFC.2011.OMT5 . hal-00585463

\section{HAL Id: hal-00585463 https://hal.science/hal-00585463}

Submitted on 13 Apr 2011

HAL is a multi-disciplinary open access archive for the deposit and dissemination of scientific research documents, whether they are published or not. The documents may come from teaching and research institutions in France or abroad, or from public or private research centers.
L'archive ouverte pluridisciplinaire HAL, est destinée au dépôt et à la diffusion de documents scientifiques de niveau recherche, publiés ou non, émanant des établissements d'enseignement et de recherche français ou étrangers, des laboratoires publics ou privés. 


\title{
All-optical phase-preserving amplitude regeneration of 28- Gbaud RZ-DQPSK signals with a microcavity saturable absorber in a recirculating loop experiment
}

\author{
Quang Trung Le ${ }^{1,2}$, Laurent Bramerie ${ }^{1,2}$, Mathilde Gay ${ }^{1,2}$, Michel Joindot ${ }^{1,2}$, Jean-Claude Simon ${ }^{1,2}$ \\ ${ }^{1}$ Université Européenne de Bretagne, France \\ ${ }^{2}$ CNRS Foton (UMR 6082), Enssat, BP 80518, 22305 Lannion cedex, France. \\ laurent.bramerie@enssat.fr \\ Arthur O'Hare \\ School of Physics, Dublin Institute of Technology, Dublin, Ireland \\ Hoang Trung Nguyen, Jean-Louis Oudar \\ CNRS-LPN, Route de Nozay, F-91460 Marcoussis, France
}

\begin{abstract}
Microcavity Saturable absorber is used for phase-preserving amplitude regeneration of RZ-DQPSK signals. The regenerator is assessed in a 28 Gbaud recirculating loop showing system tolerance to nonlinear phase noise and distance improvement.

OCIS codes: (060.2330) Optical communications; (190.5970) Semiconductor nonlinear optics
\end{abstract}

\section{Introduction}

Phase-encoded modulation formats, such as return-to-zero differential quadrature phase-shift keying (RZ DQPSK), have recently attracted much attention for 100G Ethernet systems and this has led to significant research [1]. The reach of an optical phase-shift keying transmission is mainly limited by the accumulation of both linear and nonlinear phase noise. Linear phase noise results from amplified spontaneous emission (ASE) in optical amplifiers. Nonlinear phase noise (NLPN) results from intra- and interchannel nonlinearities, such as self phase modulation (SPM) and cross phase modulation (XPM) which convert amplitude noise to phase noise [2]. It is clear that by increasing the launched channel power, the impact of the linear phase noise can be reduced. However, in this case, the nonlinear phase noise (NLPN) becomes the major impairment factor.

In the literature, it is shown that phase-preserving amplitude regeneration would be an attractive solution to enhance the phase-encoded system performance [3]. By reducing the amplitude noise, which is the origin of NLPN, phase-preserving amplitude regenerators prevent the accumulation of NLPN during transmission. The cascadability of the phase-preserving amplitude regenerators based on four-wave mixing in fibre [4] and on nonlinear amplifier loop mirror [5] have ben experimentally demonstrated. Both use short-pulse RZ DPSK signals at $10 \mathrm{Gbit} / \mathrm{s}$.

Recently, we have shown that a novel microcavity saturable absorber allowing power stabilization (SA1) [6] can provide phase-preserving amplitude noise reduction in a DPSK system [7]. The cascadability of this device in a DPSK system has been demonstrated [8]. Compared to other phase-preserving regenerators, the microcavity saturable absorber provides the advantage of a simple, compact and WDM compatible solution (regeneration of several tens of WDM channel on less than $1 \mathrm{~mm}^{2}$ ) [9].

In this paper, we assess for the first time the efficiency of all-optical phase-preserving amplitude regeneration in a DQPSK system. The SA1-based regenerator is concatenated in a recirculating loop at 28 Gbaud. Different regeneration spans are studied to find a better trade-off between the gain provided by regeneration and the device insertion loss.

\section{Demonstration of SA1-regeneration effect}

Before system experiments, we perform a first experiment demonstrating the effect of the regenerator. We emulate a link with a regenerator located where only linear phase noise is present $\left(1^{\text {st }}\right.$ transmission section). By strongly reducing the amplitude noise, the SA1 prevents the creation and accumulation of NLPN in the next transmission span ( $2^{\text {nd }}$ transmission section).

The experimental setup is presented in Fig. 1. The DQPSK signal, at $1549 \mathrm{~nm}$, is generated by a nested MachZehnder modulator (MZM) with $2^{9}-1$ bit length pseudorandom binary sequence (PRBS). A second MZM is sinusoidally modulated at $28 \mathrm{GHz}$ to convert 28 Gbaud NRZ DQPSK to $50 \%$ RZ DQPSK. The $1^{\text {st }}$ transmission section is emulated by an OSNR degradation obtained by an ASE source followed by a 3-nm band-pass optical filter, centred at the working wavelength followed by an optical attenuator (ATT). 


\section{OMT5.pdf}

The signal is then boosted to $15 \mathrm{dBm}$ by EDFA1 before being sent to SA1 via an optical circulator. The $2^{\text {nd }}$ transmission section is composed of $100 \mathrm{~km}$ of non-zero dispersion-shifted fibre (NZ DSF) with chromatic dispersion of $4.5 \mathrm{ps} / \mathrm{km} / \mathrm{nm}$ at $1550 \mathrm{~nm}$, followed by a dispersion-compensating fibre (DCF). EDFA2 is used to increase the launched power up to $12.5 \mathrm{dBm}$ (necessary to generate a high enough amount of NLPN in $100 \mathrm{~km}$ ), a more realistic launch power is used in recirculating loop experiment with several amplification spans. EDFA3 compensates the residual loss. The preamplified DQPSK receiver consists of a fibre-based delay-line interferometer (DLI) and balanced detectors. The BERT is programmed to detect the correct incoming bit stream.

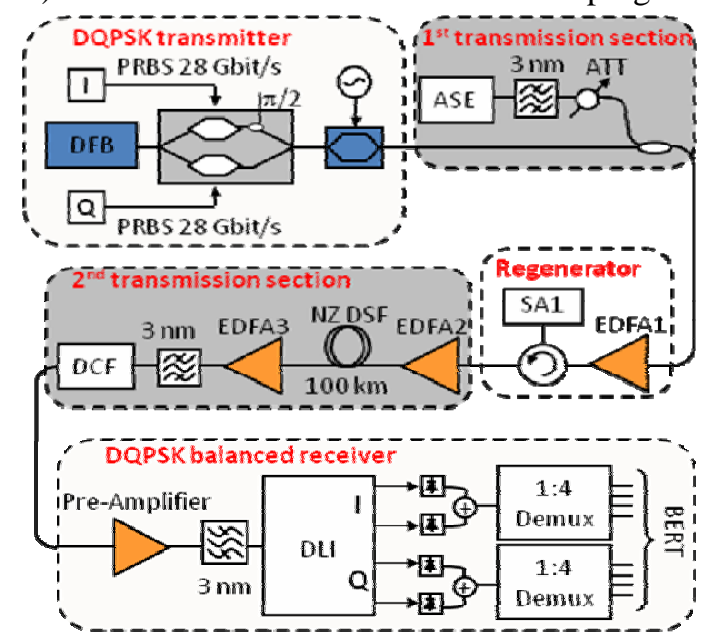

Fig. 1: Experimental setup.

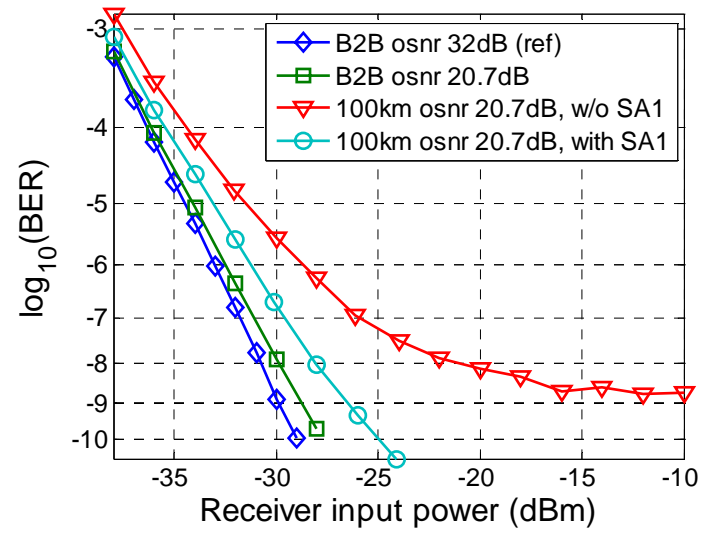

Fig. 2: The BER versus receiver input power.

Fig. 2 shows the measured BER versus the receiver input power. The first curve (diamonds) relates to our backto-back (B2B) reference with an OSNR of $32 \mathrm{~dB}$. The square-mark curve refers to B2B with an OSNR of $20.7 \mathrm{~dB}$. The BER in this case is limited by the linear phase noise induced by the ASE source. The triangle-mark curve refers to a transmission case with $12.5 \mathrm{dBm}$ launched power and without SA1. A large power penalty is obtained and an error floor at BER of $2.10^{-9}$ appears owing to linear and NLPN. When the SA1 based amplitude regenerator is used, a considerable improvement of the BER for the same fibre launch power is obtained (circle-mark curve). Indeed, the BER curve with regeneration is close to the one limited only by linear phase noise, and the error floor disappears from the measurable range of BER. The efficiency of the regenerator is thus demonstrated.

\section{Recirculating loop experiment}

In order to assess the regenerator cascadability and performance, it is concatenated in an experiment using a recirculating loop (Fig. 3). Tx and Rx are the transmitter and the receiver respectively, working at $1549 \mathrm{~nm}$.

The amplification span is composed of $100 \mathrm{~km}$ of NZ-DSF, followed by a DCF. Fibre loss is compensated for by erbium amplification. A SA1-based phase-preserving amplitude regenerator is inserted in the transmission line every $\mathrm{M}$ amplification spans. In this work, because of the limited number of available amplifiers, we consider only $\mathrm{M=1}$ and 2.

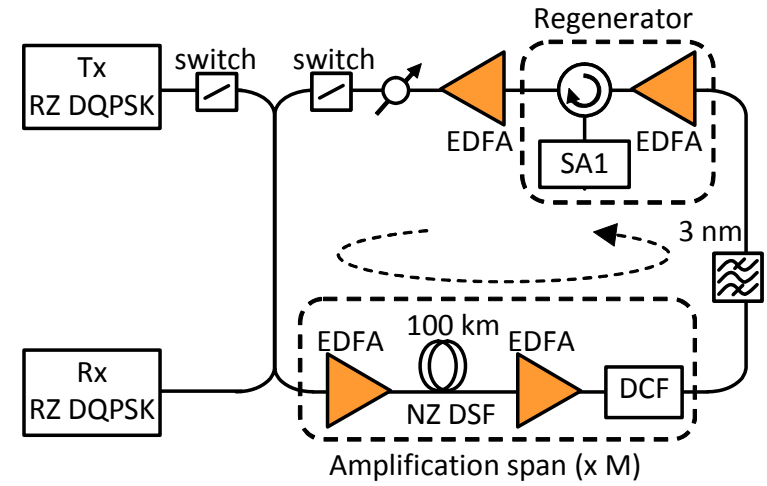

Fig. 3: Recirculating loop setup.

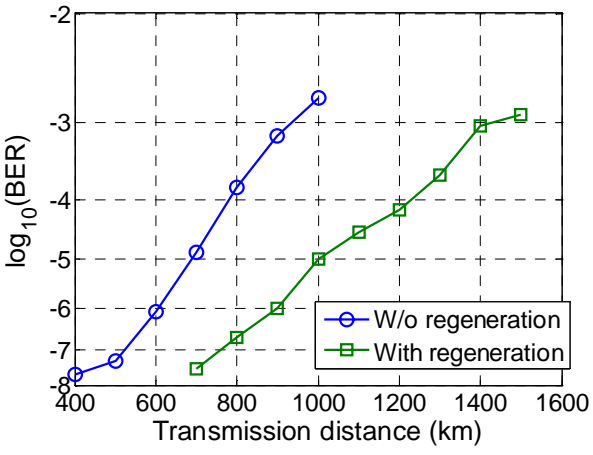

Fig. 4: BER evolution versus transmission distance w/o and with regeneration. 


\section{OMT5.pdf}

\section{Results}

Fig. 4 shows the measured BER versus transmission distance with and w/o SA1 in the case of $M=1$. The fibre launched power is set at $6 \mathrm{dBm}$. Without SA1, the BER grows rapidly due to linear and NLPN accumulation. When the phase-preserving amplitude regenerator is used, the amplitude noise is reduced and the NLPN is partly removed. As a consequence, the transmission distance is enhanced thanks to the regenerator. A transmission distance of $1200 \mathrm{~km}$ is reached for a BER of $10^{-4}$ compared to $800 \mathrm{~km}$ without regeneration.

In order to evaluate more accurately the distance improvement brought by SA1, we optimize the fibre launched power for both cases with and w/o it. BER is measured versus transmission distance for different launched powers, as depicted in Fig. 5, where the iso-BER curves associated with $B E R=10^{-n}, n=3,4,5$ and 6 are drawn. For a given BER, the distance increases first with the launched power in the linear regime (OSNR increases with the launched power), reaches a maximum. Then the transmission distance decreases with the launched power because nonlinear effects become the main degradation source and degrade more and more severely the system performance. Let us consider the particular iso-BER curve for $\mathrm{BER}=10^{-4}$ as depicted in Fig.6 (dash line), the same iso-BER curve w/o regenerator is also plotted (solid line). Without regeneration, the maximum transmission distance corresponds to a launched power of $2 \mathrm{dBm}$. When the SA1-based regenerator is used, the largest transmission distance is multiplied by a factor of 1.2 and occurs at $5 \mathrm{dBm}$ instead of $2 \mathrm{dBm}$. This reveals a significant improvement of system tolerance to the nonlinear effect.

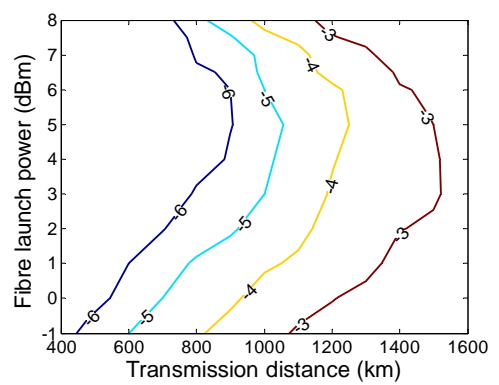

Fig. 5: BER (log scale) with concatenated SA1 versus launch power and transmission distance

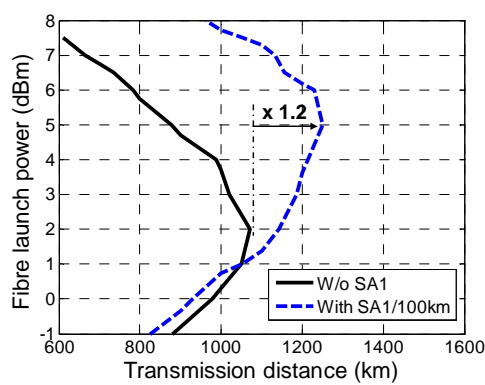

Fig. 6: Iso-BER curves (BER=10-4) w/o and with regeneration every $100 \mathrm{~km}$.

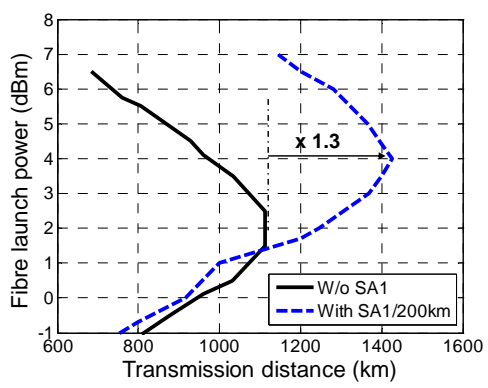

Fig. 7: Iso-BER curves (BER=10-4) w/o and with regeneration every $200 \mathrm{~km}$.

In the linear transmission regime, at low launch power, the regenerator does not bring any improvement as it preserves the signal phase. Moreover, in this case, because of its high insertion loss, the SA1 slightly degrades the OSNR.

Let us now consider the case of $\mathrm{M}=2$ where the number of amplification spans is doubled. Consequently, for the same transmission distance, the number of concatenated regenerators is reduced by a factor of 2. Fig. 7 shows the iso-BER curves for the cases with and w/o regeneration loop. Whereas in the reference case, we remain almost unchanged, the performance of the regenerated case is better (distance improvement factor of 1.3). This can be explained by the fact that by reducing the number of regenerators, a better trade-off between amplitude regeneration effect and the device insertion loss can be found. Experiment will be completed by using a path switchable loop in order to define precisely the best regeneration spacing.

\section{Conclusions}

We have reported for the first time the efficiency of phase-preserving amplitude regeneration using a microcavity saturable absorber in an RZ DQPSK transmission system at 28 Gbaud. The tolerance to the nonlinear effect of the regenerated system is enhanced, and the transmission distance is improved thanks to the regenerator. A distance improvement ratio of 1.3 is obtained with regeneration every $200 \mathrm{~km}$.

\section{References}

[1] P. Winzer et al., J. Lightw. Technol., 26, (20) 3388-3402, 2008.

[2] J. Gordon and L. Mollenauer, Optics Letters, 15, 1351-1353, 1990.

[3] S. Boscolo et al., J. Quantum Electron., 42, (7) 619-624, 2006.

[4] Matsumoto, Proc. ECOC'06, Tu1.3.5, 2006.

[5] Stephan et al., Proc. ECOC'09, 3.3.5, 2009.

[6] H. T. Nguyen et al., Appl. Phys. Lett., 92, 111107, 2008.

[7] Q. T. Le et al., Proc. OFC’10, OMT4, 2010.

[8] Q. T. Le et al., Proc. ECOC'10, Mo.2.F.3, 2010

[9] L. Bramerie et al., Proc. OFC’07, PDP1, 2007. 